Service social

\title{
Application d'une approche de médiation en protection de la jeunesse : qu'en pensent les intervenants ?
}

\author{
Sylvie Drapeau, Julie Tremblay, Geneviève Lessard, Daniel Turcotte, Gilles \\ Mireault et Marie-Hélène Gagné
}

Volume 60, numéro 2, 2014

URI : https://id.erudit.org/iderudit/1027988ar

DOI : https://doi.org/10.7202/1027988ar

Aller au sommaire du numéro

\section{Éditeur(s)}

École de service social de l’Université Laval

ISSN

1708-1734 (numérique)

Découvrir la revue

Citer cet article

Drapeau, S., Tremblay, J., Lessard, G., Turcotte, D., Mireault, G. \& Gagné, M.-H. (2014). Application d'une approche de médiation en protection de la jeunesse : qu'en pensent les intervenants ? Service social, 60(2), 14-28.

https://doi.org/10.7202/1027988ar

\section{Résumé de l'article}

La présente étude qualitative vise à documenter l'expérience d'intervenants de la protection de la jeunesse qui appliquent une approche de médiation. Cette approche repose sur l'identification des besoins de tous les acteurs concernés par une situation et sur le choix d'options susceptibles de mener à un projet d'entente satisfaisant pour toutes les parties. Quatre groupes de discussion ont été organisés dans les trois centres jeunesse participants pour un total de 33 intervenants ( 25 femmes et 8 hommes). L'analyse du discours des participants permet de décrire : 1 ) les effets perçus de l'approche de médiation sur les processus d'intervention et sur les familles; 2) les enjeux reliés à l'application de cette approche; 3 ) les caractéristiques de la clientèle plus propices à l'application de l'approche. 


\title{
Application d'une approche de médiation en protection de la jeunesse : qu'en pensent les intervenants ?
}

\author{
DRAPEAU, Sylvie \\ TREMBLAY, Julie \\ LESSARD, Geneviève \\ TURCOTTE, Daniel \\ MIREAULT, GILLES \\ GAGNÉ, MARIE-HÉLÈNE
}

\section{RÉSUMÉ}

La présente étude qualitative vise à documenter l'expérience d'intervenants de la protection de la jeunesse qui appliquent une approche de médiation. Cette approche repose sur l'identification des besoins de tous les acteurs concernés par une situation et sur le choix d'options susceptibles de mener à un projet d'entente satisfaisant pour toutes les parties. Quatre groupes de discussion ont été organisés dans les trois centres jeunesse participants pour un total de 33 intervenants ( 25 femmes et 8 hommes). L'analyse du discours des participants permet de décrire : 1) les effets perçus de l'approche de médiation sur les processus d'intervention et sur les familles; 2) les enjeux reliés à l'application de cette approche; 3) les caractéristiques de la clientèle plus propices à l'application de l'approche.

Mots clés: Médiation, protection de la jeunesse, approche consensuelle, implication parental, participation des parents.

\begin{abstract}
This qualitative study aims to document the experience of youth protection's workers who applies a mediation approach. This approach relies on identifying needs of all participants concerned by a situation and on choosing options that could lead to a draft agreement satisfactory to all of them. Four focus groups were held in the three participating youth centers including a total of 33 workers ( 25 women and 8 men). The discourse analysis of the participants brings to light 1) the perceived effects of mediation approach on the intervention process and the families, 2) the issues related to the application of this approach and 3) the families' characteristics more favorable for using the approach.
\end{abstract}

Keywords: Mediation, youth protection, parent's participation, parent's involvement. 


\section{INTRODUCTION}

En 2006, le gouvernement du Québec a apporté des modifications substantielles à la Loi sur la protection de la jeunesse (LPJ). Entre autres, la nouvelle loi affirme que toutes les interventions auprès d'un enfant et de ses parents doivent «privilégier, lorsque les circonstances sont appropriées, les moyens qui permettent à l'enfant et à ses parents de participer activement à la prise de décision et au choix des mesures qui les concernent » (art. 2.3, LPJ). Cette disposition réclame une plus grande participation des parents dans la résolution de leurs difficultés, favorisant ainsi le recours à des approches de médiation (Dumais, 2012).

Au cours des dernières années, trois centres jeunesse (Québec-Institut universitaire, Chaudière-Appalaches et Montérégie) se sont associés pour expérimenter une approche de médiation s'appuyant sur la négociation sur «intérêts ». Cette approche repose sur l'identification des besoins de tous les acteurs concernés par une situation et sur le choix d'options susceptibles de mener à un projet d'entente satisfaisant pour toutes les parties. Elle part d'une volonté d'établir un nouveau cadre d'intervention qui place l'enfant et sa famille au cœur de la prise de décision. Le présent article porte sur l'implantation de cette approche selon le point de vue des intervenants qui l'ont appliquée. Après avoir situé le paradigme d'intervention sous-jacent à ce modèle, nous présentons le cadre méthodologique de la recherche pour aborder ensuite le point de vue des intervenants qui ont participé à la recherche.

\section{Un nouveau paradigme d'intervention}

Dans les milieux de pratique sociale, on reconnaît assez facilement que certains éléments des modèles traditionnels peuvent nuire à l'engagement des enfants et des parents. La notion de déficit (qui est associée à un manquement grave aux obligations parentales ou à une contravention aux règles ou aux normes sociales), qui se lit en filigrane des décisions stipulant que la sécurité ou le développement d'un enfant est compromis, entraîne souvent une disqualification des parents. Paradoxalement, et alors qu'ils sont encore sous le choc d'un verdict de compromission, les intervenants sociaux leur demandent de collaborer en participant à l'élaboration d'un plan d'intervention. À la différence des approches centrées sur les problèmes, l'approche de médiation évite de s'engager dans une dynamique de disqualification. Elle cherche plutôt à mobiliser le jeune et ses parents autour de l'identification de leurs besoins, tout en reconnaissant leurs forces, leurs compétences et leurs ressources.

La médiation est généralement définie comme un processus volontaire de négociation auquel participe un tiers neutre et impartial qui ne détient pas l'autorité d'imposer des solutions, mais qui a le mandat d'aider les parties à résoudre leurs conflits de façon consensuelle (Maresca, 1995 ; Marcotte et al., 1997 ; Wall, Stark et Standifer, 2001). Elle s'inscrit dans le courant des approches alternatives de résolution de conflit.

L'approche utilisée en protection de la jeunesse s'inspire des méthodes développées en médiation familiale (Filion, 1998). Elle offre une structure de résolution de problèmes et de communication coopérative qui repose sur la présence simultanée des parties en conflit et mise sur la valorisation des compétences des acteurs en présence (Marcotte et Cyr, 2002). 
L'approche de médiation accorde une place centrale aux acteurs dans les orientations à prendre : c'est à eux qu'il revient de déterminer les changements à apporter pour assurer la sécurité et le développement de l'enfant. Dans l'application de cette approche, tous les acteurs (parents, enfants, intervenants et autres personnes significatives) sont invités à partager leur vision de la situation, à exprimer leurs besoins et à proposer des moyens pour résoudre leurs problèmes.

\section{Les particularités du modèle utilisé en centre jeunesse}

L'approche de médiation utilisée au Québec en protection de la jeunesse présente deux particularités qui la distingue des approches utilisées dans le reste du Canada et aux États-Unis. Premièrement, elle n'est pas appliquée par des services externes à la protection de la jeunesse, mais est plutôt intégrée aux services courants d'intervention auprès des jeunes et des familles. La participation dans le processus de médiation ne fait donc pas l'objet d'un choix explicite par les jeunes et les parents, bien qu'ils soient informés de l'approche consensuelle qui sera appliquée. Par contre, si à un moment ou à un autre, les membres de la famille refusent de coopérer, l'intervenant poursuit le processus habituel d'évaluation ou d'intervention puisqu'il prend place dans un contexte non volontaire. Deuxièmement, les techniques de médiation sont appliquées par un intervenant social qui n'agit pas comme un tiers impartial, mais comme un acteur détenant un certain pouvoir décisionnel puisqu'il a la responsabilité d'assurer la protection de l'enfant. À ce titre, il détient un certain pouvoir et il doit répondre à l'obligation de saisir le tribunal si aucune entente n'intervient et que la sécurité ou le développement de l'enfant demeure compromis.

\section{Effets et enjeux des programmes de médiation}

Les études sur les approches de médiation mettent en lumière plusieurs effets positifs, notamment un niveau élevé de collaboration et d'engagement dans la recherche de solutions (Thoennes, 2009 ; Bryan et al., 2011), un retour plus rapide des enfants dans leur milieu familial (Pringle, 2003 ; Gatowski et al., 2005) et une plus grande satisfaction à l'égard des services reçus (Bryan et al., 2011). Ces résultats tiendraient au fait que les plans de services développés dans le cadre d'une démarche de médiation seraient plus créatifs et mieux adaptés aux besoins spécifiques de chacune des familles que ceux développés dans le cadre des approches traditionnelles (Thoennes, 1997 ; Gatowski et al., 2005). Les études qui documentent le point de vue des intervenants montrent aussi des taux de satisfaction élevés face à cette approche (Gatowski, Dobbin et Litchfield, 2001 ; Litchfield et al., 2003 ; Cunningham et van Leeuwen, 2005 ; Bryan et al., 2011). Elle apparaît donc prometteuse sur le plan des bénéfices à la fois pour la clientèle des centres jeunesse et pour les intervenants. Elle n'est toutefois pas exempte d'enjeux, particulièrement au Québec où les intervenants doivent concilier la position de médiateur et leur rôle de délégué du Directeur de la protection de la jeunesse (DPJ).

Un de ces enjeux concerne le partage du pouvoir, condition essentielle à l'engagement actif des parents et à leur implication dans la prise de décision. Des recherches montrent que, malgré leurs idéaux et leurs valeurs, il peut être difficile pour les intervenants d'instaurer un tel partage dans le contexte d'une évaluation ou d'un suivi découlant d'allégations de mauvais traitement 
envers l'enfant (Saint-Jacques et al., 2006 ; Ney, Stoltz et Maloney, 2013). La ligne est mince entre le partage des préoccupations vis-à-vis de la sécurité de l'enfant et l'imposition des mesures pour assurer sa protection. Le contexte non volontaire de l'intervention en protection de la jeunesse et les contraintes organisationnelles des centres jeunesse contribuent à complexifier le rôle de l'intervenant qui mise sur la participation active des parents.

Dans la même veine, l'application d'une approche de médiation suscite des inquiétudes quant à la défense du meilleur intérêt de l'enfant. La recherche d'une entente viable et satisfaisante pour toutes les parties se fait-elle au détriment de l'intérêt de l'enfant ? De ces interrogations légitimes ont découlé plusieurs écrits balisant la pratique et précisant que la protection de l'enfant est le but non négociable de l'intervention (Pryor et Boyce, 2005). II demeure que l'application d'approches de médiation place l'intervenant dans un rôle qui implique de concilier la compréhension des besoins des parents et l'écoute de leurs solutions avec la correction des conditions qui compromettent la sécurité ou le développement de l'enfant (Stanley et al., 2003). Selon Dobbin, Gatowski et Litchfield (2001), il s'agit d'un des principaux défis que doivent relever les intervenants qui appliquent ce genre d'approches.

En somme, si l'application des approches de médiation en contexte d'autorité paraît prometteuse, elle comporte aussi des enjeux importants pour les intervenants. Le présent article vise à enrichir les connaissances sur ce sujet en documentant l'expérience d'intervenants de la protection de la jeunesse qui appliquent une approche de médiation.

\section{Questions de recherche}

Cet article présente une analyse qualitative des propos des intervenants tenus lors de groupes de discussion abordant les grandes questions suivantes : selon la perception des intervenants, quels sont les avantages et les inconvénients de l'approche de médiation sur les processus d'intervention et la clientèle ? Quels sont les enjeux reliés à l'application de cette approche dans leur contexte? Dans quelle mesure cette approche s'applique-t-elle aux différentes problématiques rencontrées ? Les résultats présentés constituent un volet d'une recherche plus vaste visant à évaluer l'implantation et les effets d'une approche de médiation auprès de jeunes suivis en vertu de l'article 38 de la LPJ.

\section{MÉTHODOLOGIE}

\section{Brève description de l'intervention appliquée}

L'appellation approche de médiation est utilisée dans ce projet pour décrire une méthode de traitement des situations de jeunes et de familles en difficulté, qui s'inspire des principes de la médiation. Les intervenants, qui sont formés à l'approche de médiation dans le cadre du programme national de formation (PNF), misent sur le processus et les techniques de la médiation bien qu'ils ne soient pas des médiateurs accrédités au sens du Règlement sur la médiation familiale.

L'application de cette approche implique un processus clinique en trois étapes (Module GPJ3, PNF, 2010). La première étape, l'accueil, est axée sur l'information. Elle vise à clarifier 
auprès de l'usager et de sa famille le mandat et le rôle du DPJ, le but de la rencontre ainsi que le processus clinique de médiation utilisé par l'intervenant. La deuxième étape, la négociation selon les intérêts, se divise en deux sous-étapes : 1) le décodage et la validation des besoins de chacune des parties impliquées et l'établissement des besoins communs ; 2) la recherche et la sélection d'options susceptibles de répondre aux besoins communs, jugés prioritaires. Elle implique la tenue d'au moins une rencontre regroupant tous les acteurs familiaux pendant laquelle un « tableau des besoins » est complété et partagé avec les membres de la famille. La troisième étape, le projet d'entente, sert à exposer les faits démontrant que la sécurité ou le développement de l'enfant est compromis, les besoins identifiés et les options choisies par les différents acteurs pour répondre aux besoins jugés prioritaires. À chacune de ces étapes, les intervenants sont invités à rédiger divers rapports qui laissent des traces de leur démarche.

Dans le cadre du présent projet, deux services ont été ciblés pour appliquer l'approche de médiation auprès des parents, soit l'Évaluation/Orientation et l'Application des mesures. Selon le processus d'intervention en protection de la jeunesse, l'évaluation de la situation survient lorsqu'un signalement est retenu en vertu de la LPJ. Cette évaluation porte sur la vérification des faits signalés et sur l'analyse de la situation de l'enfant en fonction de sa vulnérabilité et de la capacité de ses parents et de son milieu d'assurer sa sécurité et son développement. Lorsque ceux-ci semblent compromis, le DPJ prend la situation de l'enfant en charge et décide de son orientation. Cette dernière englobe le choix du régime, volontaire ou judiciaire, ainsi que le choix des mesures nécessaires pour mettre fin à la situation et pour éviter qu'elle se reproduise. Suite à l'orientation, les usagers sont dirigés vers le service d'application des mesures où un intervenant social met en place les mesures prévues (Manuel de référence sur la protection de la jeunesse, 2010).

\section{Population à l'étude et échantillon}

Les participants à ce projet devaient répondre à trois critères : 1) occuper une fonction d'agent de relations humaines dans le cadre de la LPJ ; 2) avoir reçu depuis au moins deux mois la formation de trois jours du PNF sur l'approche de médiation ; et 3) avoir appliqué cette approche dans au moins un de leur dossier. L'objectif était de réunir des répondants qui avaient une expérience concrète, sans pour autant être des experts de l'approche. Les intervenants qui correspondaient à ces critères ont été invités à participer de manière volontaire.

Quatre groupes de discussion ont été organisés dans les trois centres jeunesse participants. Chaque groupe a réuni entre 7 et 9 intervenants pour un total de 33 (25 femmes et 8 hommes). Ces intervenants travaillaient dans les secteurs de l'évaluation/orientation $(36,4 \%)$ ou de l'application des mesures (63,6 \%). Le groupe d'âge le plus représenté $(39,4 \%)$ était celui des 25 à 34 ans. Près des deux tiers (63,6 \%) possédaient une formation en travail social. Les participants avaient été formés à l'approche de médiation depuis 30 mois en moyenne. Le nombre moyen d'années d'expérience dans un centre jeunesse était de 11 ans.

\section{Procédure de collecte des données}

Les données ont été collectées à l'aide d'entrevues de groupe en s'appuyant sur les techniques d'animation des focus group (Simard, 1989 ; Krueger, 1994) qui prévoient la présence d'un 
animateur et d'un observateur. Les informations ont été collectées à l'aide d'un guide de discussion composé de huit questions ouvertes portant notamment sur les thèmes suivants : 1 ) effets observés sur la clientèle et sur les processus d'intervention ; 2) expérience en lien avec son application (rôle de l'intervenant, place des acteurs dans le processus), notamment en lien avec les diverses problématiques rencontrées en protection de la jeunesse. Enfin, un questionnaire a été remis à chacun des participants afin de recueillir des données sociodémographiques. La durée des entrevues de groupe fut d'environ deux heures.

\section{Stratégie d'analyse des données}

Avec l'accord écrit des participants, le contenu des discussions a été enregistré et retranscrit intégralement sous la forme de verbatims. Le matériel a été traité à l'aide de la méthode d'analyse de contenu thématique (L'Écuyer, 1990 ; Paillé et Mucchielli, 2008). L'analyse a été réalisée par deux des auteurs de l'article en utilisant le logiciel N'Vivo comme support à cette analyse. La grille d'analyse a été élaborée selon un modèle mixte dont les grandes catégories de départ correspondaient aux thèmes de l'entrevue. Des sous-thèmes ont émergé à partir des propos des participants. Des échanges entre les auteurs, sous la forme d'allers-retours, ont permis d'affiner la grille d'analyse. Les thèmes ont ensuite été discutés avec quelques acteurs impliqués dans l'implantation de l'approche de médiation de chacun des trois centres jeunesse participants (Directeurs de la protection de la jeunesse, gestionnaires et intervenants).

\section{RÉSULTATS}

\section{Les effets perçus de l'approche sur les processus d'intervention et la clientèle}

De façon générale, les intervenants sont satisfaits de l'application de l'approche de médiation. Ils y associent des mots tels que consensus, écoute, mobilisation, respect et responsabilisation auxquels ils attribuent une valeur positive. L'avantage qui fait le plus consensus concerne la qualité de la relation avec les parents. En effet, au dire des répondants, l'approche de médiation crée un climat de travail positif en améliorant la collaboration et la communication avec les membres de la famille. Cette approche favorise aussi la création plus rapide d'un lien de confiance entre les parties. La diminution des conflits et des situations antagonistes facilite le travail des intervenants en leur évitant d'être confrontés à la colère et à l'agressivité de certains parents : «Pour moi, l'approche de médiation, ça m'évite de faire face à de l'agressivité » (no 4). D'experts ou d'enquêteurs, les intervenants passent à un rôle de conciliateur, ce qui leur enlève un poids des épaules. L'approche de médiation permettrait aux intervenants de faire leur travail « de façon moins menaçante » (no 1) et d'être moins axés sur le contrôle et la surveillance: «Je trouve que c'est sain pour nous, l'approche de médiation, parce que ça nous permet de lâcher prise » (no 2). Parler de « besoins » plutôt que de problèmes apaise les antagonismes et favorise une communication plus ouverte entre les parties.

Un deuxième avantage concerne la collaboration avec les partenaires qui interviennent dans le dossier de la famille. À ce propos, des répondants soulignent que l'application de l'approche de médiation, plus précisément le fait de se centrer sur les besoins de l'enfant, favorise la collaboration intersectorielle en évitant les positions rigides reliées à la mission des 
établissements. La réalité des familles et la complexité de leurs besoins sont alors au cœur des discussions entre partenaires, ce qui amène à chercher collectivement des solutions afin de soutenir les parents. Des répondants soulignent aussi que leur passage au Tribunal est facilité quand ils centrent leur propos sur les besoins des enfants, plutôt que sur la démonstration de l'incapacité des parents. Ils ont l'impression que leur témoignage est moins contesté. Comme il leur est plus naturel de s'exprimer de cette façon, leur crédibilité s'en trouve accrue : « On sent une plus grande écoute de la part des juges et des avocats parce qu'on est centré sur les besoins, et on est beaucoup moins contesté » (no 3).

Aucun participant ne trouve, à proprement parler, d'effets négatifs à l'approche. Ils sont en accord avec la philosophie et les valeurs qui y sont véhiculées : « On ne peut pas être contre la vertu » (no 1). Cela dit, les bénéfices identifiés par la majorité sont nuancés par certains répondants qui émettent des réserves en raison des enjeux de cette approche, surtout dans un contexte d'appropriation d'une nouvelle pratique. Ce grand thème, qui fait l'objet de la prochaine section, se subdivise en trois sous-thèmes: le rôle de l'intervenant, l'appropriation d'une nouvelle pratique et les contraintes liées au contexte de travail.

\section{Les enjeux reliés à l'application de l'approche}

\section{Le rôle de l'intervenant}

Les discussions portant sur le rôle de l'intervenant sont animées, car tous ne partagent pas le même point de vue sur ce sujet. En détenant le mandat d'assurer la protection de l'enfant, les intervenants sont « une partie prenante au dossier » (no 3); ils ne peuvent être totalement neutres et impartiaux. Bien que reconnaissant cette situation, certains n'y voient pas un obstacle car ils se perçoivent comme des « animateurs » du processus de médiation. Ces répondants considèrent qu'ils sont en mesure de prendre un recul par rapport à la situation et d'établir une neutralité sur le plan «émotif »; ils ne sont toutefois pas neutres face au résultat puisque leur but demeure la protection de l'enfant. D'autres ont l'impression d'occuper une position ambiguë : «C'est toujours un rôle ambigu» (no 2): «Quand on embarque dans un processus de médiation, on doit être ouvert aux options qui vont être choisies. [...] Sinon c'est pire. On donne le pouvoir au client et on reprend le pouvoir. C'est comme si on jouait avec eux » (no 3).

Pour tous les répondants, il est clair que l'enfant est au centre du processus de médiation et que ses besoins demeurent la priorité : «Mon travail c'est ça. II faut que ce parent-là réponde aux besoins de cet enfant-là » (no 3). Ils se perçoivent comme «le défenseur des besoins de l'enfant » (no 1), que ce dernier soit présent ou non aux rencontres. Ce rôle leur paraît d'autant plus important lorsque l'enfant est en bas âge et qu'ils ont l'impression d'être sa seule voix. Et, comme l'approche de médiation est axée sur la reconnaissance des besoins des parents, plutôt que sur leur déficit, certains participants ont l'impression de se retrouver entre deux positions difficiles à concilier. En se centrant sur les besoins des parents, certains se demandent si ceux de l'enfant seront négligés. Ces participants craignent de moins bien jouer leur rôle de protection en omettant des informations importantes, surtout lors de l'évaluation. Questionnentils suffisamment les parents sur les faits ayant mené à leur signalement et à la compromission de l'enfant? 
Des répondants soupèsent aussi les besoins des enfants et ceux des parents et admettent qu'en faisant passer les besoins du jeune au premier plan, ils prennent le risque de briser la relation qu'ils ont bâtie avec les parents: "Si je dois protéger l'enfant, on doit à un moment donné répondre à ce besoin-là en priorité sur le besoin du parent. C'est sûr que des fois ça coupe une relation » (no 3). Certains répondants mentionnent que la transparence sur le mandat du DPJ est la clé pour sortir de cette impasse et préserver la relation avec le parent.

\section{L'appropriation d'une nouvelle pratique}

Si certains sont d'avis que l'approche de médiation repose d'abord sur un « savoir-être », sur une philosophie qui s'intègre à la pratique courante, d'autres croient plutôt que le savoir-être doit être combiné à une application stricte du processus et des techniques de l'approche pour obtenir les bénéfices escomptés : «Faire le processus au complet, avec toutes les étapes, où tout est blanc ou noir, ce n'est pas la réalité » (no 2) ; "C'est devenu dans ma pratique, dans mon quotidien. Les concepts, je les applique presque de façon systématique. J'ai toujours dans ma serviette mon petit tableau et je l'utilise dans mes rencontres » (no 3).

Est-ce que toutes les étapes doivent être franchies ? Est-ce que toutes les exigences doivent être remplies (tableau des besoins, traces dans les rapports écrits, etc.) ? La rencontre familiale doit-elle nécessairement avoir lieu ? « C'est ça la question, est-ce qu'il faut vraiment les asseoir ensemble? » (no2).

Les exigences de l'établissement, les différents niveaux d'aisance avec cette approche, la cohérence des messages, tous ces éléments font en sorte que les intervenants ne s'entendent pas sur les éléments essentiels à respecter pour prétendre à une application véritable de l'approche de médiation ${ }^{1}$.

\section{Les contraintes liées au contexte de travail}

Le contexte de travail ressort comme un obstacle à l'implantation de cette approche pour plusieurs répondants. Entre autres, il leur apparaît difficile de mener à bien l'ensemble du processus dans le temps qui leur est imparti, surtout quand ils ressentent une pression de performance. Leurs propos suggèrent que l'approche de médiation exige plus de temps que l'approche traditionnelle. C'est pourquoi certains intervenants, notamment parmi ceux du service Évaluation/Orientation, décident de ne pas l'appliquer. Certains sont aussi d'avis que leur charge de travail est trop grande pour pouvoir appliquer l'approche dans tous leurs dossiers: "C'est notre contexte de travail qui va au désavantage de l'approche. Il faudrait qu'ils nous libèrent de la moitié de notre charge pour qu'on l'applique et ça n'arrivera jamais » (no1).

Cet écart dans les pratiques des deux services introduit une discontinuité que déplorent certains répondants. Certaines familles peuvent rencontrer un intervenant formé à la médiation lors de l'application des mesures, mais pas lors de l'évaluation (ou inversement). Les

\footnotetext{
${ }^{1}$ Au moment de l'étude, aucun guide de pratique n'était disponible et les lignes directrices n'étaient pas encore très formalisées dans les établissements, ce qui explique probablement l'accent mis sur ces questions.
} 
répondants déplorent les incohérences que cela produit pour les familles et leurs difficultés à assurer un processus clinique harmonieux: "On dirait que j'ai de la misère à enligner un dossier avec l'approche de médiation quand ça n'a pas été fait comme ça à l'évaluation » (no1).

\section{Les caractéristiques de la clientèle}

Questionnés sur l'applicabilité de l'approche, les intervenants insistent sur l'importance du timing et du contexte: "C'est vraiment à la pièce, dans le contexte, avec les personnes » (no1). Certaines caractéristiques de la clientèle semblent plus propices à l'application de l'approche. Entre autres, le fait que les parties soient «volontaires » ou reconnaissent minimalement le problème facilite l'utilisation de l'approche : «On n'en viendra jamais à faire un processus de médiation avec un gun sur la tempe. Ça prend des gens qui ont un minimum de vouloir et de reconnaissance »(no2). Également, il est plus facile d'appliquer l'approche de médiation avec une clientèle ayant la capacité de s'impliquer dans un processus d'intervention. Cela demande que leurs besoins de base soient minimalement satisfaits et qu'ils aient des habiletés de communication. L'application de l'approche auprès de personnes présentant un problème de santé mentale non stabilisé ou une déficience intellectuelle apparaît plus complexe en raison de leurs limites cognitives ou de leur difficulté à se centrer sur les besoins des autres. Les participants ne sont toutefois pas unanimes à ce sujet : « Je ne suis pas tout à fait d'accord. [...] Le fait qu'ils n'aient pas de capacité, ça ne veut pas dire qu'ils n'ont pas de pouvoir sur la décision. Ça ne veut pas dire qu'ils ne vont pas participer » (no3).

Les situations de conflits de séparation et d'aliénation parentale soulèvent encore plus de questionnements. Pour certains, la médiation est l'approche idéale dans ce contexte, car elle permet de sortir des impasses, alors que, pour d'autres, c'est l'inverse : ces situations sont trop complexes et rigidifiées pour se prêter à l'exercice. Une des difficultés rencontrées par ces participants est l'exigence de réunir toutes les parties impliquées afin qu'elles partagent leurs points de vue. Selon certains répondants, la rencontre familiale exige du temps de préparation et susciterait la méfiance de certains clients : «Des fois, ça cause problème pour certains clients de tous les asseoir ensemble. Beaucoup de résistance. Il a fallu que je sois flexible » (no1). Ils remettent donc en question la pertinence de cette exigence, d'autant plus que, selon eux, faire circuler l'information entre les membres de la famille permet d'atteindre aussi bien les objectifs.

Le discours des intervenants fait également ressortir une mise en garde à propos de l'utilisation de l'approche dans des situations familiales où il y a une forme de violence conjugale ou d'abus de pouvoir: «Ça donne des munitions au client sur un angle plus négatif dépendamment s'il tourne ça à son avantage » (no2). Les intervenants qui utilisent l'approche dans ces situations précisent devoir prendre le temps de bien préparer «le terrain » en rencontrant les parents individuellement et en préparant soigneusement la rencontre familiale.

L'implication des enfants dans le processus de médiation a également été abordée par les répondants. À ce sujet, il ne semble pas y avoir de règle universelle: «la ligne n'est pas parfaite » (no4). Il apparaît important de tenir compte du contexte familial et du développement affectif de l'enfant. Cependant, dans le cas des adolescents, la participation fait l'unanimité. L'adolescent a acquis une maturité qui lui permet d'exprimer ses besoins devant son parent: 
«Ce n'est pas un enfant, on n'a pas à le préserver, c'est un adolescent » (no1). II serait également facile de les impliquer dans le processus de médiation : «Les ados en trouble de la conduite sont super contents que l'on fasse de la médiation parce que l'on arrête de leur taper sur la tête et on regarde les choses qui sont positives » (no4). Ces derniers feraient d'ailleurs preuve d'une plus grande ouverture que lors des interventions habituelles.

\section{DISCUSSION}

L'objectif de cet article était de décrire la perception des intervenants sociaux à propos de l'application d'une approche de médiation en contexte de protection de la jeunesse. Trois questions ont été explorées: quels sont les avantages et les inconvénients de l'approche de médiation sur les processus d'intervention et la clientèle ? Quels sont les enjeux reliés à l'application de cette approche dans le contexte de la protection de la jeunesse ? Dans quelle mesure cette approche s'applique-t-elle aux différentes problématiques rencontrées ?

En lien avec la première question, les intervenants ont mentionné plusieurs avantages à cette pratique, notamment une amélioration de la relation avec les parents, une plus grande participation des clients au processus d'intervention de même qu'une plus grande motivation à changer la situation de compromission. Les intervenants apprécient la collaboration et la qualité de la communication établies avec les membres de la famille. L'approche de médiation leur permet d'aborder les « vrais » enjeux, de mieux comprendre les besoins des clients et de leur faire davantage confiance. Bien que la pratique au Québec soit différente de celle au Canada ou aux États-Unis, ces résultats s'inscrivent dans la lignée de ce qui a été observé dans les écrits du domaine (Litchfield et al., 2003 ; Colman et Ruppel 2007 ; Bryan et al., 2011 ; Gladstone et al., 2012).

Dans le contexte de la protection de la jeunesse, la collaboration avec les parents n'est pas acquise, les intervenants peuvent être confrontés à la méfiance, à la colère et au déni de la part des parents qui ont peur de perdre leurs enfants et sont obligés de participer à des services qu'ils n'ont pas demandés, services qui ne correspondent pas nécessairement aux besoins qu'ils ressentent. L'approche de médiation favorise une plus grande ouverture de la part des parents et un meilleur partage du pouvoir, ce qui est central pour l'engagement du parent dans le processus d'intervention (Saint-Jacques et al., 2006 ; De Boer et Coady, 2007).

Les résultats de cette étude font aussi ressortir le paradoxe qui peut exister entre le soutien aux parents et la protection de l'enfant (Chamberland et Durning, 2009). Il peut exister une tension entre ces rôles et les intervenants doivent constamment agir dans des situations complexes où les besoins et les intérêts des uns et des autres évoluent dans un équilibre délicat (Duffey, Taylor et McCall, 2006). Exercer ce double rôle peut s'avérer difficile (Stanley et al., 2003) et entraîner une confusion, voire des dérives éthiques, selon Barsky (2007).

Les préoccupations exprimées à propos de la sécurité de l'enfant pourraient expliquer pourquoi cette pratique fait toujours l'objet d'une certaine controverse même si elle est de plus en plus répandue dans le système de la protection de la jeunesse au Canada et aux États-Unis (Colman et Ruppel, 2007 ; Edwards, 2009). Pour atténuer les inquiétudes, des guides sont désormais disponibles pour baliser les pratiques et diverses mesures ont été mises sur pied, 
notamment la participation d'un avocat affecté à l'enfant, le recours à des médiateurs chevronnés et la révision à la Cour de tous les éléments de l'entente (Edwards, 2009).

Une autre source de questionnement provient de l'application de l'approche de médiation dans des situations de conflits de séparation, d'aliénation parentale ou de violence conjugale. Ce résultat fait écho aux études réalisées sur la clientèle de la protection de la jeunesse, mais aussi, de manière plus large, aux études sur les pratiques de médiation familiale (Kathol, 2009). Les préoccupations principales concernent la sécurité des victimes et la capacité de toutes les parties en cause à participer pleinement au processus de médiation. Si, pour certains auteurs, cette pratique n'est pas appropriée lorsque le déséquilibre des rapports de force est trop grand, d'autres suggèrent d'y recourir mais en adoptant des mesures complémentaires comme inviter un consultant/expert à se joindre aux rencontres, par exemple un représentant dans le domaine de la violence conjugale (Giovannucci et Largent, 2009), ou appliquer un protocole spécifiquement adapté à ce genre de situations (Edwards, 2009).

Finalement, la question de la participation des enfants au processus de médiation a aussi été soulevée. Selon les propos recueillis, l'âge minimum requis pour participer à ce genre de rencontre n'est pas clairement défini. Ce constat semble partagé par les auteurs du domaine qui proposent différentes stratégies pour respecter le droit de participation des enfants, tout en les protégeant (Edwards, 2009 ; Giovannucci et Largent, 2009). Par exemple, il est suggéré de les rencontrer individuellement pour écouter leurs besoins et s'en faire les porte-parole, plutôt que de les mettre en présence des autres parties. Dans d'autres cas, il est suggéré de préparer soigneusement les enfants afin de faciliter leur implication lors des rencontres ou de les structurer de façon à ce que les enfants ne soient conviés qu'à certains moments pertinents.

\section{CONCLUSION}

Le présent article a mis en lumière le point de vue d'intervenants ayant appliqué une approche de médiation dans le cadre de leur mandat de protection de la jeunesse. Trois centres jeunesse (Québec-Institut universitaire, Chaudière-Appalaches et Montérégie) se sont associés pour rendre ce projet possible. Il a nécessité une grande ouverture et une collaboration étroite entre tous les partenaires impliqués.

Cette étude montre que les intervenants sont favorables à cette approche et y voient plusieurs avantages, notamment sur le plan de la relation avec les parents. Cela dit, appliquer une approche de médiation dans le contexte de la protection de la jeunesse représente un défi important puisque l'intervenant est appelé à jouer le rôle d'animateur du processus de médiation tout en conservant le mandat de protection de l'enfant. Selon les propos recueillis dans le cadre de cette étude, ce défi est possible à relever, mais il s'agit d'un changement important de pratique nécessitant une période d'appropriation et des stratégies d'accompagnement organisationnelles.

Pour être cohérents avec les principes de l'approche, les intervenants doivent éviter le piège de la « pré-définition » du problème et être très francs avec les parents à propos de leur rôle et de leur mandat (Healy et Darlington, 2009). Ils doivent apprendre un nouveau langage et développer de nouvelles habiletés, c'est pourquoi il est recommandé que des stratégies 
d'accompagnement soient mises en place au-delà des formations proprement dites (Marcotte et al., 1997; Cunningham et van Leeuwen, 2005 ; Eaton, Whalen et Anderson, 2007).

Rappelons que les données de cette étude ont été recueillies pendant la période d'expérimentation de l'approche, ce qui implique que des changements dans la pratique des intervenants étaient en cours; certains étaient plus à l'aise et plus expérimentés, alors que d'autres en étaient à leurs premiers essais. Les informations recueillies offrent donc un portrait circonscrit dans le temps. Un guide de pratique est désormais disponible pour les intervenants désireux d'appliquer cette approche dans les centres jeunesse (Chouinard et al., 2013).

DRAPEAU, Sylvie

Professeure

École de psychologie

Université Laval

TREMBLAY, Julie

Professionnelle de recherche

Centre jeunesse de Québec

Institut universitaire

LESSARD, Geneviève

Professeure

École de service social

Université Laval

TURCOTTE, Daniel

Professeur titulaire

École de service social

Université Laval

MIREAULT, GILLES

Chercheur

Centre jeunesse de Québec

Institut universitaire

GAGNÉ, MARIE-HÉLÈNE

Professeure titulaire

École de psychologie

Université Laval 


\section{BIBLIOGRAPHIE}

Association des centres jeunesse du Québec (2010). Module générique en protection de

la jeunesse (GPJ 3). Approche de médiation en protection de la jeunesse. Montréal: Association des centres jeunesse du Québec.

Barsky, A.E. (2007). « Mediative evaluations: The pros and perils of blending roles », Family Court Review, vol. 45, $n^{\circ} 4$, p. 560-572.

Bryan, V., C. Collins-Camargo, N. Anderson et C. Kantar (2011). «Making the Case for Evaluation Partnerships in Child Protection Mediation Programs: Insights from a Case Study », Juvenile and Family Court Journal, vol. 62, n² 2, p. 35-52.

Chamberland, C., et P. Durning (2009). « La violence et la maltraitance envers les enfants : un enjeu social et scientifique », Santé, Société et Solidarité, vol. 8, n 1, p. 5-11.

Chouinard, D., É. Dionne, S. Drapeau et J. Tremblay (2013). «L'approche de médiation en contexte de protection de la jeunesse », Guide de pratique réalisé par les Centres jeunesse de Québec-Institut universitaire, de la Montérégie et de Chaudière-Appalaches.

Colman, R., et J. Ruppel (2007). Child Permanency Mediation Pilot Project. Multi-Site Process and Outcome Evaluation Study, New York State Office of Children \& Family Services, 56 p.

Cunningham, A., et J. van Leeuwen (2005). Finding the third option: The experience of the London child protection mediation project, Center for Children and Families in the Justice System, London Family Court Clinic, Inc., 214 p.

De Boer, C., et N. Coady (2007). « Good helping relationships in child welfare: learning from stories of success », Child and Family Social Work, vol. 12, n 1, p. 32-42.

Dobbin, S.A., S.I. Gatowski et M. Litchfield (2001). The Essex County Child Welfare Mediation Program: Evaluation Results and Recommendations, National Council of Juvenile and Family Court Judges.

Duffy, J., B. Taylor et S. McCall (2006). «Human Rights and Decision-making in Child Protection through Explicit Argumentation », Child Care in Practice, vol. 12, n², p. 81-95.

Dumais, J. (2012). « Philosophie des lois de protection de la jeunesse et protection de l'enfance au Québec et en France », dans P. Brisson et M. Savourey-Alezra, Protection de l'enfance et de la jeunesse-médiation. Comment accompagner la famille autrement. L'approche médiation: Principes et méthodologie, Québec, Presses de l'Université Laval, p. 51-77.

Eaton, M., P. Whalen et G. Anderson (2007). « Permanency Planning Mediation Pilot Program: the Michigan Experience », The Michigan Child Welfare Law Journal, vol. 10, $n^{\circ}$ 3, p. 2-10.

Edwards, H.L. (2009). «Child Protection Mediation: A 25-year perspective », Family Court Review, vol. 47, n 1, p. 69-80.

Filion, L. (1998). «L'approche médiation en contexte d'autorité », Document de formation non publié. Montréal, Centre jeunesse de Montréal - Institut universitaire. 
Gatowski, S.I., S.A. Dobbin et M. Litchfield (2001). The Miami Model Court Family Decision-Making Conference Program: Evaluation Results, Permanency Planning for Children Department of the National Council of Juvenile and Family Court Judges, 89 p.

Gatowski, S.I., S.A. Dobbin, M. Litchfield et J. Oetjen (2005). Mediation in Child Protection Cases: An Evaluation of the Washington, D.C., family Court Child Protection Mediation Program, National Council of Juvenile and Family Court Judges, 27 p.

Giovannucci, M., et K. Largent (2009). « A guide to effective child protection mediation: Lessons from 25 years of practice », Family Court Review, vol. 47, n 1, p. 38-52.

Gladstone, J., G. Dumbrill, B. Leslie, A. Koster, M. Young et A. Ismaila (2012). « Looking at engagement and outcome from the perspectives of child protection workers and parents », Children and Youth Services Review, vol. 34, $\mathrm{n}^{\circ}$ 1, p. 112-118.

Healy, K., et Y. Darlington (2009). « Service user participation in diverse child protection contexts: principles for practice », Child and Family Social Work, vol. 14, n 4, p. 420-430.

Kathol, J. (2009). « Trends in Child Protection Mediation : Results of the Think Tank Survey and Interviews », Family Court Review, vol. 47, n 1, p. 116-128.

Krueger, R.A. (1994). Focus groups: A practical guide for applied research, Thousand Oaks (CA), Sage Publications, 255 p.

L'Écuyer, R. (1990). Méthodologie de l'analyse développementale de contenu. Méthode GPS et concept de soi, Sillery, Presses de l'Université du Québec, 472 p.

Litchfield, M., J.A. Oetjen, D.M. Maxwell, S.I. Gatowski et S.A. Dobbin (2003). Empowering Families in Child Protection Cases: an Implementation Evaluation of Hawai'i's 'Ohana Conferencing Program, Reno (NV), National Council of Juvenile and Family Court Judges, 88 p.

Manuel de référence sur la protection de la jeunesse (2010). Gouvernement du Québec, ministère de la Santé et des Services sociaux. <http://publications.msss.gouv.qc.ca/acrobat/f/documentation/2010/10-838-04.pdf $\geq$.

Marcotte, R., P. Brisson, J. Olivier et M.K. Laflamme (1997). Évaluation du processus de médiation en protection de la jeunesse, Lévis, Centres jeunesse Chaudière-Appalaches.

Marcotte, R., et F. Cyr (2002). Évaluation de la formation approche-médiation (vol. 1), Centre jeunesse de Montréal - Institut universitaire.

Maresca, J. (1995). « Mediating child protection cases », Child Welfare, vol. 74, nº 3, p. 731-742.

Ney, T., J.-A. Stoltz et M. Maloney (2013). « Voice, power and discourse: Experiences of participants in family group conferences in the context of child protection », Journal of Social Work, vol. 13, n², p. 184-202.

Paillé, P., et A. Mucchielli (2008). L'analyse qualitative en sciences humaines et sociale. $2^{\mathrm{e}}$ édition. Paris, Armand Collin. 
Pringle, J. (2003). Evaluation of the Surrey Court Project: Facilitated Planning Meeting, Final Report, Vancouver, British Columbia Ministry of Attorney General.

Pryor, D. et S. Boyce (2005). The Center for Dispute Settlement Child Permanency Mediation Program. A survey of Stakeholders and Key Program Participants, Center for Government Research.

Saint-Jacques, M.-C., S. Drapeau, G. Lessard, A. Beaudoin (2006). «Parent involvement practices in child protection - A matter of know-how and attitude », Child and Adolescent Social Work Journal, vol. 23, n² 2, p. 196-215.

Simard, G. (1989). Animer, planifier et évaluer l'action. La méthode du «focus group », Laval, Mondia, $102 \mathrm{p}$.

Stanley, N., B. Penhale, D. Riordan, R.S. Barbour et S. Holden (2003). «Working on the interface : Identifying professionnal responses to families with mental health and child-care needs », Health and Social Care in the Community, vol. 11, n³, p. 208-218.

Thoennes, N. (1997). « An Evaluation of Child Protection Mediation in Five California Courts », Family and Conciliation Courts Review, vol. 35, n² 2, p. 184-195.

Thoennes, N. (2009). «What We Know Now: Findings from Dependency Mediation Research », Family Court Review, vol. 47, $\mathrm{n}^{\circ} 1$, p. 21-37.

Wall, J.A., J.B. Stark et R.L. Standifer (2001). « Mediation. A current review and theory development », Journal of Conflict Resolution, vol. 45, n³, p. 370-391. 